\title{
Effects of Entrainment and Mixing on the Wegener-Bergeron-Findeisen Process
}

\author{
FABIAN HOFFMANN \\ Cooperative Institute for Research in Environmental Sciences, University of Colorado Boulder, and \\ Chemical Sciences Laboratory, NOAA/Earth System Research Laboratories, Boulder, Colorado
}

(Manuscript received 16 October 2019, in final form 21 April 2020)

\begin{abstract}
The growth of ice crystals at the expense of water droplets, the Wegener-Bergeron-Findeisen (WBF) process, is of major importance for the production of precipitation in mixed-phase clouds. The effects of entrainment and mixing on WBF, however, are not well understood, and small-scale inhomogeneities in the thermodynamic and hydrometeor fields are typically neglected in current models. By applying the linear eddy model, a millimeter-resolution representation of turbulent deformation and molecular diffusion, we investigate these small-scale effects on WBF. While we show that entrainment is accelerating WBF by contributing to the evaporation of liquid droplets, entrainment may also cause aforementioned inhomogeneities, particularly regions filled with exclusively ice or liquid hydrometeors, which tend to decelerate WBF if the ice crystal concentration exceeds $100 \mathrm{~L}^{-1}$. At lower ice crystal concentrations, even weak turbulence can homogenize hydrometeor and thermodynamic fields sufficiently fast so as to not affect WBF. Independent of the ice crystal concentration, it is shown that a fully resolved entrainment and mixing process may delay the nucleation of entrained aerosols to ice crystals, thereby delaying the uptake of water vapor by the ice phase, further slowing down WBF. All in all, this study indicates that, under specific conditions, small-scale inhomogeneities associated with entrainment and mixing counteract the accelerated WBF in entraining clouds. However, further research is required to assess the importance of the newly discovered processes more broadly in fully coupled, evolving mixed-phase cloud systems.
\end{abstract}

\section{Introduction}

The Wegener-Bergeron-Findeisen (WBF) process is considered the main microphysical pathway to precipitation in mixed-phase clouds (Wegener 1911; Bergeron 1935; Findeisen 1938). Its physical basis is the difference in the water vapor saturation pressure over ice and liquid water, resulting in a net flow of water molecules from the liquid to the ice phase, potentially causing the depletion of all liquid water and the accelerated fallout of ice crystals. Due to its direct effect on the production of precipitation, the WBF process has significant implications for the global radiation budget by limiting cloud lifetime and cloud cover, but also by affecting the partitioning of water into the ice and liquid phase with commensurate effects on the cloud albedo (e.g., Storelvmo et al. 2015; Korolev et al. 2017). Distinct partitioning of ice and liquid water is observed on scales from several kilometers to tens of meters

\footnotetext{
Corresponding author: Fabian Hoffmann, fabian.hoffmann@ noaa.gov
}

(e.g., Chylek and Borel 2004), but the distribution of these phases on smaller scales is not well understood. These scales are predominantly affected by entrainment and mixing, and according to Korolev et al. (2017), "Until now no studies on entrainment and mixing in mixed-phase clouds have been conducted." Because of this lack of understanding, the default assumption for modeling mixed-phase clouds is that liquid droplets and ice crystals are well mixed on unresolved scales; that is, there are no regions in which one hydrometeor species occurs preferentially.

Is this assumption reasonable? This can be understood from the Damköhler number,

$$
\mathrm{Da}=\frac{\tau_{\text {mix }}}{\tau_{\text {react }}},
$$

where $\tau_{\text {mix }}$ is the turbulent mixing time scale and $\tau_{\text {react }}$ a to-be-defined reaction time scale of a microphysical process (e.g., Baker and Latham 1979). During WBF, ice crystals and liquid droplets change the thermodynamic properties of the air surrounding them: ice crystals create a water-subsaturated environment due to their 
more efficient diffusional growth, while liquid droplets tend to counteract these water subsaturations by evaporation. If the turbulent stirring is faster than the microphysical reaction $(\mathrm{Da} \ll 1)$, all hydrometeors will experience the same homogeneous thermodynamic environment. If, however, the turbulent stirring is slower than the microphysical reaction $(\mathrm{Da} \gg 1)$, the inhomogeneous spatial distribution of the hydrometeors and the thermodynamic fields surrounding them matters, creating regions in which WBF is reduced (or even inhibited) due to the absence of ice crystals or liquid droplets. The length scale at which these inhomogeneities start to matter is obtained by the turbulent mixing time scale,

$$
\tau_{\text {mix }}=\left(\frac{l^{2}}{\epsilon}\right)^{1 / 3},
$$

which estimates the time for a flow feature of length scale $l$, for example, an entrained segment of environmental air, to break down to the Kolmogorov length scale, assuming a kinetic energy dissipation rate $\epsilon$. By setting Da $=1$, Lehmann et al. (2009) showed that the transition from inhomogeneous to homogeneous mixing takes place at

$$
l_{\text {trans }}=\epsilon^{1 / 2} \tau_{\text {react }}^{3 / 2},
$$

that is, the length scale at which $\tau_{\text {react }}=\tau_{\text {mix }}$. For all smaller length scales, the assumption of homogeneity is valid. Accordingly, models with a resolution finer than $l_{\text {trans }}$ can neglect variations in the spatial distribution of hydrometeors on unresolved scales since these do not feedback onto the microphysical processes due to sufficiently fast turbulent mixing. However, this length scale depends on the microphysical processes considered and their corresponding microphysical reaction time scale $\tau_{\text {react }}$.

The microphysical reaction time scale that describes how fast super- or subsaturations are depleted by condensation/evaporation or deposition/sublimation is termed the phase relaxation time scale (e.g., Squires 1952). It may be defined as

$$
\tau_{\text {phase }, \varphi} \approx\left(4 \pi D_{v} N_{\varphi} c \bar{r}_{\varphi}\right)^{-1},
$$

where $\varphi$ indicates the liquid $(\varphi=l)$ or ice phase $(\varphi=i)$, $D_{v}$ is the molecular diffusion coefficient for water vapor in air, $N_{\varphi}$ the number concentration of the respective hydrometeor species, and $\bar{r}_{\varphi}$ an average radius of all liquid or ice hydrometeors. The factor $0<c \leq 1$ depicts the normalized capacitance of the hydrometeors, with $c=1$ for spheres (e.g., Pruppacher and Klett 1997, chapter 13.3). For the liquid phase, $\tau_{\text {phase }, 1}$ is usually on the order of $1 \mathrm{~s}$, and inhomogeneous mixing prevails on all length scales larger than typically $10 \mathrm{~cm}$ for low turbulent clouds such as stratocumuli $\left(\epsilon=10 \mathrm{~cm}^{2} \mathrm{~s}^{-3}\right.$, $\left.N_{1}=100 \mathrm{~cm}^{-3}, \bar{r}_{1}=10 \mu \mathrm{m}\right)$. For the ice phase, $\tau_{\mathrm{phase}, i}$ is several orders of magnitude larger due to the significantly smaller number of ice crystals, resulting in phase relaxation time scales between $10^{4}$ and $10^{2} \mathrm{~s}$, and a commensurate transition length scale between $10 \mathrm{~km}$ and $10 \mathrm{~m}\left(\epsilon=10 \mathrm{~cm}^{2} \mathrm{~s}^{-3}, N_{i}=1-100 \mathrm{~L}^{-1}\right.$, $\left.\bar{r}_{i}=100 \mu \mathrm{m}\right)$. Similarly, large transition length scales can be obtained for the related glaciation time scale, that is, the time necessary to transform all liquid water to ice via the WBF process:

$$
\tau_{\mathrm{gl}} \approx \frac{3}{2} \tau_{\text {phase }, i} \frac{q_{l, 0}}{q_{s, l}-q_{s, i}},
$$

where the initial liquid water mixing ratio $q_{l, 0}$ and the ice supersaturation mixing ratio at liquid-water saturation $q_{s, l}-q_{s, i}$ are typically on the order $0.1 \mathrm{~g} \mathrm{~kg}^{-1}$. [See the appendix for the derivation of (5).] Note that a positive (negative) vertical velocity increases (decreases) $\tau_{\mathrm{gl}}$ by counteracting (intensifying) the water subsaturations caused by the growing ice crystals (Korolev and Field 2008). Furthermore, the nucleation of ice crystals might also affect WBF by determining the number of ice crystals and hence the phase relaxation time scale considered in (5). Ice nucleation can be described as a time-dependent, stochastic process that is-based on the thermodynamic conditions and the physical and chemical composition of the ice nuclei (IN) — associated with a time scale $\tau_{\text {nuc }}$ between 10 and 100 s (e.g., Ervens and Feingold 2012). The associated transition length scale is therefore between 1 and $10 \mathrm{~m}$.

Finally, it is necessary to take into account the sedimentation of the considered hydrometeors that spread out a microphysical reaction over the distance fallen, homogenizing its effect especially when turbulence is low (e.g., Tölle and Krueger 2014). The associated sedimentation time scale is defined as

$$
\tau_{\text {sed }}=\frac{l}{w_{\text {sed }}},
$$

where $w_{\text {sed }}$ is the sedimentation velocity of the considered hydrometeor. We can combine $\tau_{\text {sed }}$ with the turbulent mixing time scale $\tau_{\text {mix }}$ to yield a generalized mixing time scale

$$
\tau_{\text {mix }}^{*}=\left(\tau_{\text {mix }}^{-1}+\tau_{\text {sed }}^{-1}\right)^{-1},
$$

which accounts for the effects of turbulent mixing and sedimentation.

These theoretical considerations indicate that inhomogeneous mixing can affect mixed-phase clouds; 
however, it is restricted to length scales larger than $1 \mathrm{~m}$, that is, significantly larger than the transition length scales found in pure liquid-phase clouds. However, these length scales can be smaller than the resolution of typical models used to study mixed-phase clouds. Furthermore, these length scales are also well within the range typically affected by the entrainment of environmental air into a cloud ( $\leq 100 \mathrm{~m}$; e.g., Bodenschatz et al. 2010). Therefore, the main questions for this study are as follows: How do entrainment and mixing affect the small-scale distribution of hydrometeors in a mixed-phase cloud? And how do these inhomogeneities feed back into the mixed-phase microphysical processes? We approach these questions using the linear eddy model (LEM), a one-dimensional but highly accurate representation of turbulent mixing (Kerstein 1988; Krueger 1993), coupled to a Lagrangian mixed-phase microphysics model (Ervens et al. 2011; Hoffmann et al. 2015). Foundations and necessary extensions of this modeling framework are described in section 2. Modeling results are presented in section 3 . And the study is concluded in section 4.

\section{Modeling framework}

In the following study, a cloud parcel is simulated that experiences an entrainment event. The LEM is used for an explicit representation of turbulent deformation and molecular diffusion within this parcel, while a Lagrangian approach is used for the representation of mixed-phase microphysics. Since the LEM and Lagrangian microphysics have been described in great detail already, only the new or relevant parts for this study will be summarized in the following, and the reader is referred to the referenced literature for more details. [Note that the LEM is applied as a stand-alone model, and not as the subgrid-scale parameterization of a three-dimensional simulation as in previous publications of the author (Hoffmann et al. 2019; Hoffmann and Feingold 2019).]

\section{a. Lagrangian mixed-phase microphysics}

Lagrangian microphysics have been used for decades (e.g., Howell 1949), and regained interest in the last decade by the advent of superdroplet or superparticle approaches (e.g., Shima et al. 2009), in which one simulated particle represents a large number of real atmospheric particles. For this study, we combine the Lagrangian liquid-phase microphysics model by Hoffmann et al. $(2015,2017)$ with the ice-phase processes of the mixed-phase model by Ervens et al. (2011).

In the applied Lagrangian microphysical model, each simulated particle represents an individual or an ensemble of several identical hydrometeors, that is, aerosol particles, liquid droplets, or ice crystals. The simulated particle is, however, allowed to undergo transitions between these categories. The activation of liquid droplets is explicitly considered by including curvature and solute effects in the diffusional growth equation, thereby predicting changes in the wet radius of each deliquescent aerosol particle and liquid droplet, as done in the liquid-phase microphysics model of the author (e.g., Hoffmann et al. 2015). As in the mixed-phase model of Ervens et al. (2011), deposition nucleation of ice crystals is based on Chen et al. (2008). Using this nucleation rate, a stochastic approach is applied to decide if a simulated particle transforms from an aerosol to an ice crystal (Unterstrasser and Sölch 2014). Immersion freezing is not considered in this study since the entrained aerosols do not activate to cloud droplets, therefore inhibiting subsequent immersion freezing. Also identical to Ervens et al. (2011), diffusional growth of ice crystals is based on the crystal habit-predicting model by Chen and Lamb (1994), calculating the mass and aspect ratio for the main axes of a spheroid, thereby representing the ice crystal shape. Note that the applied Maxwellian diffusional growth equation inherently assumes that the hydrometeors do not influence each other, that is, distort the water vapor fields surrounding them. In reality, liquid droplets in the vicinity of an ice crystal growth might enhance its mass growth by up to $10 \%$ due to this proximity effect (e.g., Marshall and Langleben 1954; Miller and Young 1979). All possible collection processes, such as coalescence, aggregation, and riming, are neglected because they are not relevant for this study. The motion of the simulated hydrometeors is determined by sedimentation, using the terminal velocities of Beard (1976) for liquid droplets and Mitchell (1996) for ice crystals. Additionally, turbulent motions prescribed by the LEM change the location of the simulated hydrometeors as described in more detail below.

The number of identical hydrometeors represented by a Lagrangian particle is called weighting factor or multiplicity $A_{n}$ and is determined by the geometry of the LEM and the number concentrations of IN and cloud condensation nuclei $(\mathrm{CCN})$ as outlined in the next subsection in more detail. Note that the weighting factor needs to be considered for the calculation of all $\mathrm{CCN}$ - or IN-extensive properties and processes, for example, in the calculation of the ice water and liquid water mixing ratios per LEM grid box,

$$
q_{l}=\frac{1}{\rho_{a} \Delta V_{\mathrm{LEM}}} \sum_{\mathcal{N}_{\mathrm{CCN}}} A_{n} m_{n}
$$

and 


$$
q_{i}=\frac{1}{\rho_{a} \Delta V_{\mathrm{LEM}}} \sum_{\mathcal{N}_{\mathrm{IN}}} A_{n} m_{n},
$$

respectively, as well as in the effects of phase changes on the water vapor mixing ratio and temperature in each LEM grid box,

$$
\frac{d q_{v}}{d t}=-\frac{1}{\rho_{a} \Delta V_{\mathrm{LEM}}}\left(\sum_{\mathcal{N}_{\mathrm{CCN}}} A_{n} \frac{d m_{n}}{d t}+\sum_{\mathcal{N}_{\mathrm{IN}}} A_{n} \frac{d m_{n}}{d t}\right)
$$

and

$\frac{d T}{d t}=\frac{1}{c_{p} \rho_{a} \Delta V_{\mathrm{LEM}}}\left(l_{v} \sum_{\mathcal{N}_{\mathrm{CCN}}} A_{n} \frac{d m_{n}}{d t}+l_{s} \sum_{\mathcal{N}_{\mathrm{IN}}} A_{n} \frac{d m_{n}}{d t}\right)$,

respectively, where $\Delta V_{\text {LEM }}$ is the volume of an LEM grid box, $\mathcal{N}_{\mathrm{CCN}}$ and $\mathcal{N}_{\text {IN }}$ the sets of Lagrangian particles representing CCN and IN in an LEM grid box, respectively. Furthermore, $\rho_{a}$ is the density of air, $m_{n}$ is the individual water mass of the simulated hydrometeor, $c_{p}$ is the specific heat capacity of air at constant pressure, and $l_{v}$ and $l_{s}$ are the latent heat of evaporation and sublimation, respectively.

\section{b. The linear-eddy model}

The LEM is a one-dimensional representation of turbulent deformation and molecular diffusion (Kerstein 1988). Because of the LEM's reduced dimensionality, it is possible to resolve the smallest relevant length scale for turbulent mixing, the Kolmogorov length scale, while it also enables a domain large enough to include the inhomogeneities that affect mixed-phase cloud microphysics, that is, several meters or more as discussed above. However, the LEM does not predict the development of turbulence, which needs to be prescribed by choosing appropriate parameters for each simulation. Nonetheless, the LEM constitutes a valuable addition to cloud parcel simulations, which allows to include a physically based representation of turbulent entrainment and mixing (e.g., Krueger 1993; Krueger et al. 1997; Su et al. 1998).

To mimic the effects of turbulence, randomly selected segments of the LEM domain are rearranged using the triplet map that increases gradients and disperses fluid elements (Kerstein 1988). The frequency of these rearrangements and the length of the rearranged segments are based on inertial range scaling. The LEM is coupled to the Lagrangian microphysical model by the release and depletion of heat and water vapor, as well as the turbulent transport of the hydrometeors, experiencing the same rearrangements as the grid boxes of the LEM. The LEM applied here is based on the implementation by Hoffmann et al. (2019), but it has been extended to predict water vapor and temperature, instead of water supersaturation alone. The reader is referred to Kerstein (1988), Krueger (1993), and Menon and Kerstein (2011) for a more detailed description of the LEM.

The present study documents the first application of the LEM to mixed-phase microphysics. This causes a problem that is inherently absent in applications for pure liquid- or ice-phase simulations: Representing the average distance between two hydrometeors as well as the fluid volume occupied by each hydrometeor correctly. While the first property is important for studying the effects of inhomogeneities, that is, the spatial separation of ice crystals and liquid droplets, the latter property is essential to accurately represent the effects of phase changes, that is, the release and depletion of heat and water vapor. These scales can be calculated as follows.

The average distance between a pair of $\mathrm{CCN}$ or IN (including all already nucleated and activated particles, respectively) is

$$
\Delta z_{\mathrm{CCN}}=N_{\mathrm{CCN}}^{-1 / 3}
$$

and

$$
\Delta z_{\mathrm{IN}}=N_{\mathrm{IN}}^{-1 / 3},
$$

respectively, where $N_{\mathrm{CCN}}$ and $N_{\mathrm{IN}}$ are the corresponding number concentrations. Similarly, the average volume of air surrounding individual $\mathrm{CCN}$ or IN is

$$
\Delta V_{\mathrm{CCN}}=\Delta z_{\mathrm{CCN}}^{3}=N_{\mathrm{CCN}}^{-1}
$$

and

$$
\Delta V_{\mathrm{IN}}=\Delta z_{\mathrm{IN}}^{3}=N_{\mathrm{IN}}^{-1},
$$

respectively.

In the following, we will detail how these scales can be successfully represented in the LEM. The reader is also referred to Fig. 1 for an illustration of the involved scales in the LEM framework. To capture the influence of hydrometeors on the water vapor and temperature fields surrounding them, the LEM grid spacing must represent (at least) the average distance between these particles. Since $\Delta z_{\mathrm{CCN}} \ll \Delta z_{\text {IN }}$ under typical mixed-phase conditions, $\Delta z_{\mathrm{CCN}}$ is the natural choice for the vertical grid spacing of the vertically oriented LEM used in this study:

$$
\Delta z_{\mathrm{LEM}}=\Delta z_{\mathrm{CCN}} .
$$

Similarly, by placing IN with an average vertical spacing of $\Delta z_{\text {IN }}$ into the LEM, that is, several LEM grid boxes apart, this property is also represented as required. 

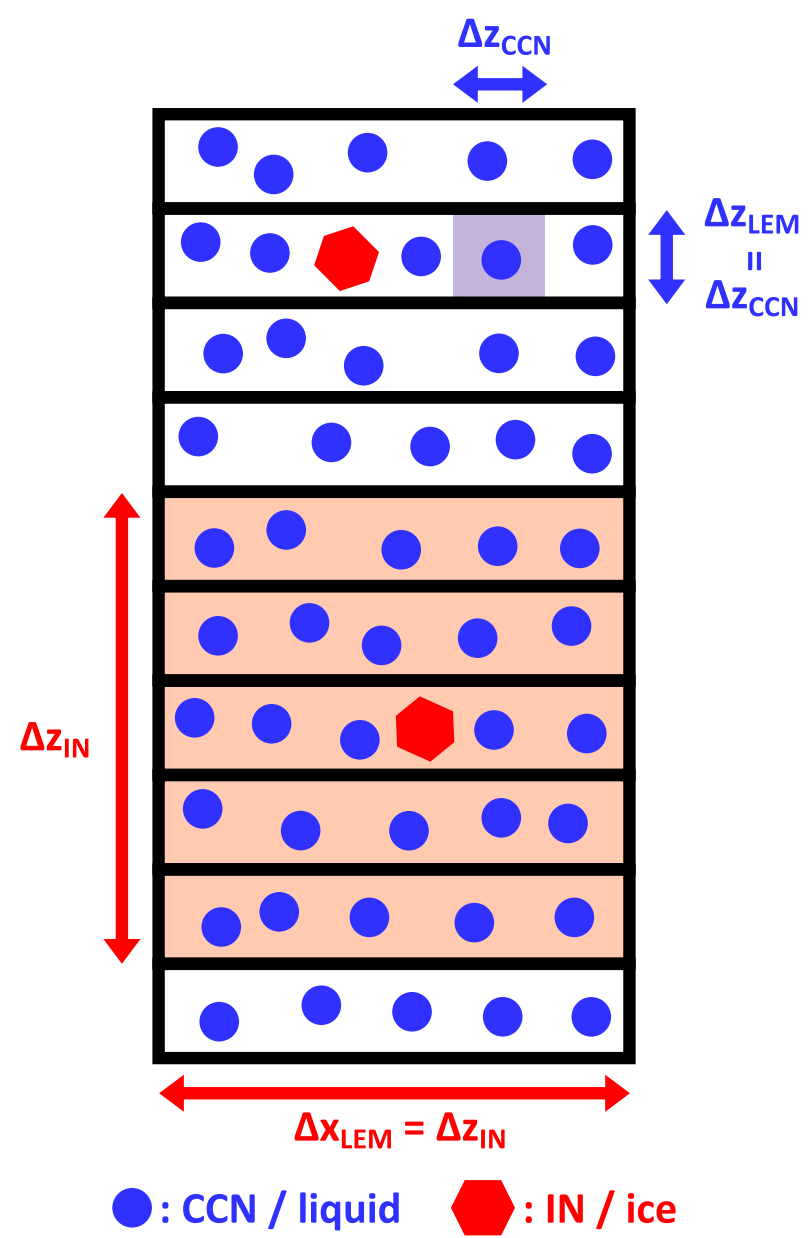

FIG. 1. Sketch illustrating the grid spacing used to set up the LEM (black lines). The vertical grid spacing is chosen to match the average distance between $\mathrm{CCN}\left(\Delta z_{\mathrm{CCN}}\right.$, blue), the horizontal grid spacing is set to match the average distance between IN $\left(\Delta z_{\mathrm{IN}}\right.$, red $)$. Due to this arrangement, not only the average distances, but also the volumes containing a liquid hydrometeor (blue-shaded squares) or an ice hydrometeor (red-shaded squares) are represented in the model.

To find one CCN in $\Delta V_{\mathrm{CCN}}$, the horizontal LEM grid spacing $\Delta x_{\text {LEM }}$ has traditionally also been assigned to $\Delta z_{\mathrm{CCN}}$. However, since IN are $\Delta z_{\mathrm{IN}}$ apart in the vertical, this choice would result in one IN in the volume $\Delta z_{\mathrm{IN}} \Delta z_{\mathrm{CCN}}^{2} \ll \Delta z_{\mathrm{IN}}^{3}=\Delta V_{\mathrm{IN}}$; that is, the number concentration of IN would be overestimated. Accordingly, the horizontal LEM grid is set to

$$
\Delta x_{\mathrm{LEM}}=\Delta z_{\mathrm{IN}},
$$

representing correctly that one IN is found in the volume $\Delta z_{\mathrm{IN}} \Delta x_{\mathrm{LEM}}^{2}=\Delta z_{\mathrm{IN}}^{3}=\Delta V_{\mathrm{IN}}$. This yields an LEM grid box volume

$$
\Delta V_{\mathrm{LEM}}=\Delta x_{\mathrm{LEM}}^{2} \Delta z_{\mathrm{LEM}}=N_{\mathrm{IN}}^{-2 / 3} N_{\mathrm{CCN}}^{-1 / 3},
$$

TABLE 1. Parameters and values used to set up the presented

\begin{tabular}{|c|c|c|c|c|c|c|}
\hline Parameter (units) & & & & Values & & \\
\hline$L(\mathrm{~m})$ & & & & 100 & & \\
\hline$\Delta z_{\text {LEM }}(\mathrm{mm})$ & & & & 2.2 & & \\
\hline$T\left({ }^{\circ} \mathrm{C}\right)$ & & & & -15 & & \\
\hline$S_{l, \mathrm{entr}}(\%)$ & & & & -5 & & \\
\hline$N_{\mathrm{CCN}}\left(\mathrm{cm}^{-3}\right)$ & & & & 100 & & \\
\hline$N_{\text {IN }}\left(\mathrm{L}^{-1}\right)$ & 0.1 & 1 & 10 & 100 & 1000 & \\
\hline $\begin{array}{l}\text { Entrained IN type } \\
\epsilon\left(\mathrm{m}^{2} \mathrm{~s}^{-3}\right)\end{array}$ & & & $10^{-5}$ & $\begin{array}{c}\text { Deposition } \\
1^{-4}\end{array}$ & $\begin{array}{c}\text { No IN } \\
10^{-3}\end{array}$ & \\
\hline$f_{\text {entr }}$ & & 0.0 & 0.1 & 0.2 & 0.4 & \\
\hline $\bar{w}\left(\mathrm{~cm} \mathrm{~s}^{-1}\right)$ & & -10 & -2 & $\mathbf{0}$ & 2 & 10 \\
\hline
\end{tabular}
simulations. Boldface values mark the base simulation.

which is much larger than $\Delta V_{\mathrm{CCN}}$. Accordingly, not only one but several CCN need to be placed in this volume to represent $\Delta V_{\mathrm{CCN}}$ correctly. The number of $\mathrm{CCN}$ is therefore determined as

$$
A_{n}=\Delta V_{\mathrm{LEM}} / \Delta V_{\mathrm{CCN}}=\left(N_{\mathrm{CCN}} / N_{\mathrm{IN}}\right)^{2 / 3} .
$$

Instead of simulating these CCN inside an LEM grid box individually, we will employ the superdroplet approach and use $A_{n}$ as the weighting factor to represent these $\mathrm{CCN}$ by a single Lagrangian particle per LEM grid box as outlined in the previous subsection. Since one IN is found in the volume $\Delta z_{\mathrm{IN}} \Delta x_{\mathrm{LEM}}^{2}=\Delta V_{\mathrm{IN}}$, the weighting factor for IN is required to be 1 .

What are the physical implications of this anisotropic LEM grid box? As desired, spatial inhomogeneities in the vertical direction can be represented on scales as small as a couple of millimeters determined by $\Delta z_{\text {LEM }}$. However, the reaction of hydrometeors to small-scale inhomogeneities is coarse-grained to an effective resolution between $\Delta z_{\mathrm{LEM}}$ and the typically larger $\Delta x_{\mathrm{LEM}}$. However, $\Delta x_{\text {LEM }}$ is typically smaller than a couple of decimeters, and therefore on scales for which the assumption of homogeneity is already valid as outlined in the introduction. In other words, the applied LEM resolves all inhomogeneities larger than a couple of decimeters successfully, and therefore the scales we expect to be affected by the small-scale flow features investigated here.

\section{c. Setup}

In the following, we will state the parameters used for setting up the base simulation. Variations of the base simulation are summarized in Table 1. All simulations use an LEM domain of $L=100 \mathrm{~m}$, cyclic in the vertical, with a grid spacing of $\Delta z_{\mathrm{LEM}}=2.15 \mathrm{~mm}$. CCN are represented by 46416 Lagrangian particles, that is, one for each LEM grid box, and a weighting factor based on (19). The number of Lagrangian particles representing IN is determined by $N_{\mathrm{IN}}$, which is varied between 
0.1 and $1000 \mathrm{~L}^{-1}$, resulting in 464 to 46416 additional Lagrangian particles placed randomly in the LEM domain. Their weighting factor is set to 1 . The time step of the model is $1 \mathrm{~s}$, but significantly reduced for processes that require subcycling (diffusional growth of liquid droplets and ice crystals; turbulent rearrangements and molecular diffusion in the LEM). The simulation duration is $600 \mathrm{~s}$.

All CCN and IN inside the cloud are assumed to be activated or nucleated. The water mass assigned to these liquid droplets and ice crystals is prescribed by a lognormal distribution with a geometric mean radius of $8 \mu \mathrm{m}$ and a geometric standard deviation of 1.36. The aspect ratio of the main ice crystal axes is initialized as 1 . After initialization, the mass change of liquid droplets and ice crystals, as well as changes in the aspect ratio of the main ice crystal axes, is calculated explicitly. For the base simulation, concentrations of $N_{\mathrm{CCN}}=100 \mathrm{~cm}^{-3}$ and $N_{\mathrm{IN}}=100 \mathrm{~L}^{-1}$ are prescribed.

The entrained aerosols constituting IN and $\mathrm{CCN}$ are prescribed as in Ervens et al. (2011) by a lognormal distribution with a geometric mean radius of $40 \mathrm{~nm}$ and a geometric standard deviation of 1.4. CCN are assumed to consist of $90 \%$ soluble ammonium sulfate. IN consist of dust with a contact angle of $10^{\circ}$, and are prescribed to nucleate by deposition. In all simulations, the concentration of the entrained $\mathrm{CCN}$ matches the in-cloud value $\left(N_{\mathrm{CCN}}=100 \mathrm{~cm}^{-3}\right)$. Similarly, the number concentration of entrained IN is set to the in-cloud value, which is $N_{\text {IN }}=100 \mathrm{~L}^{-1}$ for the base simulation. Additionally, a set of simulations without entrained IN is conducted.

The entire domain is initialized to be at water saturation at a temperature of $-15^{\circ} \mathrm{C}$ and a hydrostatic pressure of $800 \mathrm{hPa}$. For entrainment, a contiguous fraction of the domain, $f_{\text {entr }}=0.2$, is replaced by a water-subsaturated segment with $S_{l, \text { entr }}=-5 \%$ at the beginning of the simulation. The temperature of the entrained segment is not changed.

A kinetic energy dissipation rate of $\epsilon=1 \mathrm{~cm}^{2} \mathrm{~s}^{-3}$, a relatively low but still typical value for Arctic stratiform mixed-phase clouds (Shupe et al. 2008), controls the turbulent mixing calculated within the LEM, bounded by a model Kolmogorov length scale of $\eta=6 \Delta z_{\mathrm{LEM}}$ and a turbulence integral length scale of $L=100 \mathrm{~m}$, respectively. The base simulation does not exhibit a mean vertical velocity $\left(\bar{w}=0 \mathrm{~cm} \mathrm{~s}^{-1}\right)$, but stochastic vertical motions due to the LEM triplet map heat or cool parts of the LEM adiabatically. Additional simulations with mean heating or cooling rates corresponding to up- and downdrafts of \pm 10 and $\pm 2 \mathrm{~cm} \mathrm{~s}^{-1}$ are also presented.

All LEM simulations are compared to identically initialized, homogeneous box simulations, in which the mixing is instantaneous. Accordingly, we will address these two types of simulations as LEM simulations and homogeneous simulations, respectively. Further note that each simulation is repeated 10 times, using a different set of random numbers, which will alter the initial particle placement in the LEM domain, initial assignment of water and aerosol mass, LEM triplet-map rearrangements, and IN nucleation. If necessary, the results are averaged over this ensemble. This reduces the inherent stochastic noise included in each simulation.

\section{Results}

\section{a. Overview}

Figure 2 shows the temporal development of one instance of the base-case LEM simulation. The segment of water-subsaturated entrained air is initialized at $-10 \leq z \leq 10 \mathrm{~m}$ (blue shading), and is continuously mixed with the approximately water-saturated cloud (yellow and green shading). Over the entire domain, but especially apparent inside the cloud, turbulent up- and downdrafts cause intermittent signatures of super- and subsaturations, respectively. With time, the entire domain becomes water subsaturated because of the WBF process.

At the beginning of the simulation, ice crystals (black dots) and liquid droplets (not marked) are located inside the cloud, while unnucleated IN (red dots) and unactivated CCN (not marked) are placed in the entrained segment, which is devoid of any nucleated or activated hydrometeors. This ice crystal void is transported into the cloud: Turbulent mixing moves smaller chunks of entrained air into the cloud, which can be recognized by regions without ice crystals but the presence of unnucleated IN within the cloud. Additionally, the ice crystal void is also transported downward as a signature within the field of sedimenting ice crystals, falling faster than the liquid droplets. Accordingly, the void is shifted from the entrainment region downward into the cloud. Similarly, ice crystals might be located in regions devoid of any liquid droplets, for example, the first ice crystals sedimenting into the entrained segment or in regions of strong WBF evaporating all liquid droplets surrounding ice crystals, as it occurs in the end of the simulation. Naturally, these voids tend to vanish through ongoing turbulent mixing. Note that these ice crystal and liquid droplet voids will be of major interest below since they can locally inhibit the WBF process. Further note that all of these small-scale features are inherently absent in the homogeneous simulations.

For the same setup, but averaged over 10 instances, Fig. 3 shows probability distribution functions (PDFs) 


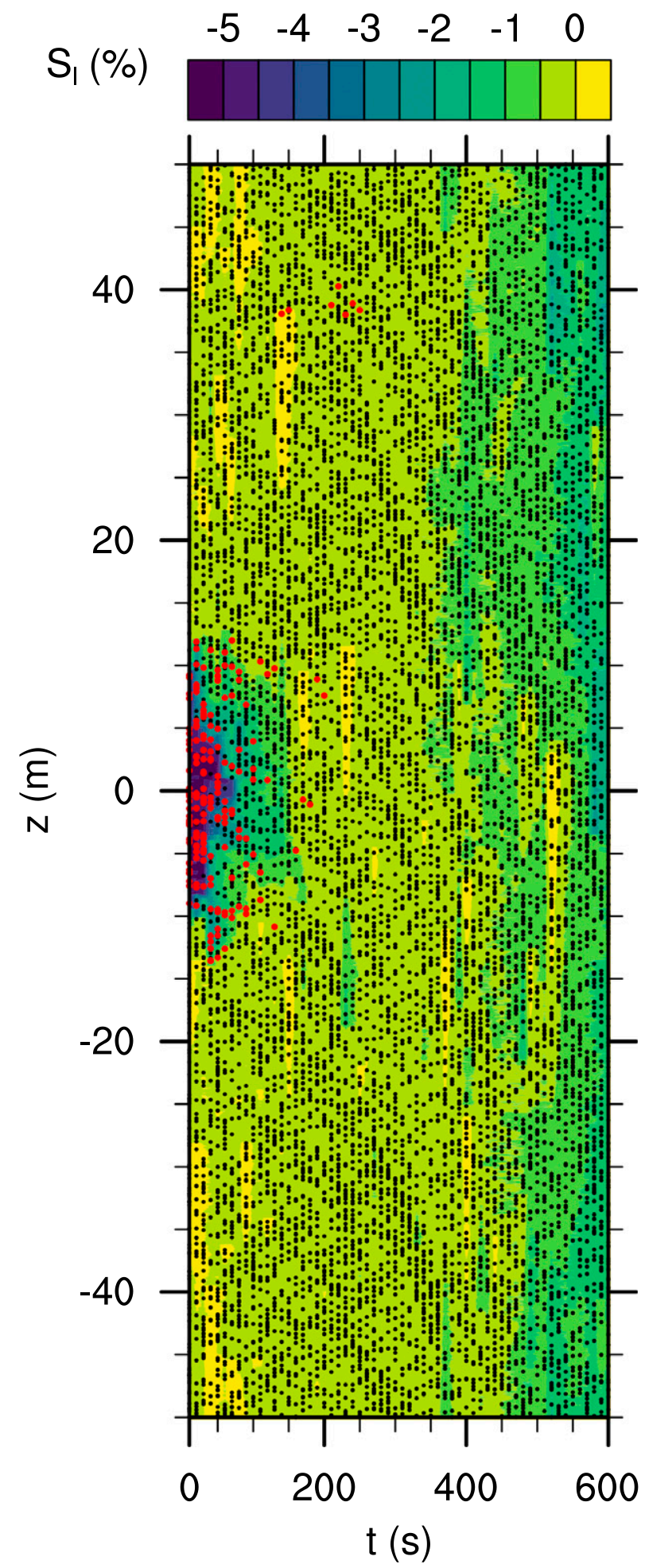

FIG. 2. Temporal development of the LEM domain for the basecase setup. Colored shading shows the liquid water supersaturation $S_{l}$, black dots are every 25 th ice crystal, red dots are every 25 th unnucleated IN. for 1) the liquid droplet radii and 2) ice crystal equivalent radii from the homogeneous simulations (dashed lines) and the LEM (continuous lines). The liquid droplets (Fig. 3a) show clearly how the initial lognormal distribution is continuously broadened to smaller sizes by evaporation, first by mixing with the entrained air and subsequently by the WBF process, which requires some ice crystal growth to be effective. Note that these two processes have distinct impacts on the droplet PDFs: The initial mixing with the entrained air is restricted to a small fraction of the model domain bordering the entrained segment. If resolved, this archetypical inhomogeneous response maintains the radii of the unaffected droplets, while it shifts the entire spectrum toward smaller radii when the mixing is forced to be homogeneous, as originally hypothesized for liquid clouds by Baker and Latham (1979). And indeed, this response is clearly visible for the largest liquid droplets $(r>30 \mu \mathrm{m})$ in the LEM, which are still present for $t \leq 200 \mathrm{~s}$, while these droplets are already evaporated to smaller radii in the homogeneous simulation. The homogeneous response of shifting the entire droplet PDF to smaller radii is also visible for the WBF process, but for both modeling approaches $(t \geq 300 \mathrm{~s})$. The reason for this is the larger transition length scale for glaciation through WBF, enabling a larger fraction of droplets to evaporate, resulting in a more homogeneous reaction.

In contrast to the previously discussed PDFs of the liquid droplet radii, the PDFs of the ice crystal equivalent radii are quickly distorted from their initial lognormal distribution, and grow toward larger radii (Fig. 3b). Initially ( $t \leq 10 \mathrm{~s})$, the ice crystals in the LEM grow faster than those in the homogeneous simulation since their bulk remains in the water-saturated cloud while all ice crystals in the homogeneous simulation experience the same, water-subsaturated environment with a commensurately lower growth rate. Afterward $(t \geq 100 \mathrm{~s})$, the LEM simulations exhibit a broader tail toward smaller radii. We will argue below that this feature results from the delayed nucleation of entrained IN in the LEM simulations as well as a reduced WBF process, slowing down the growth of some ice crystals in the LEM. At the end of the simulation, however, the PDFs of the homogeneous and LEM simulation are very similar.

To further understand the previous results, Fig. 4 shows changes in bulk quantities of the LEM (green lines) and homogeneous simulations (red lines) initialized with IN concentrations between 0.1 and $1000 \mathrm{~L}^{-1}$ (dashed patterns). The ice water mixing ratio (Fig. 4a) grows from almost negligible initial values, and increases proportional to $N_{\text {IN }}$ as long as $N_{\text {IN }}$ is smaller than $1000 \mathrm{~L}^{-1}$. For these cases, the crystal growth is 

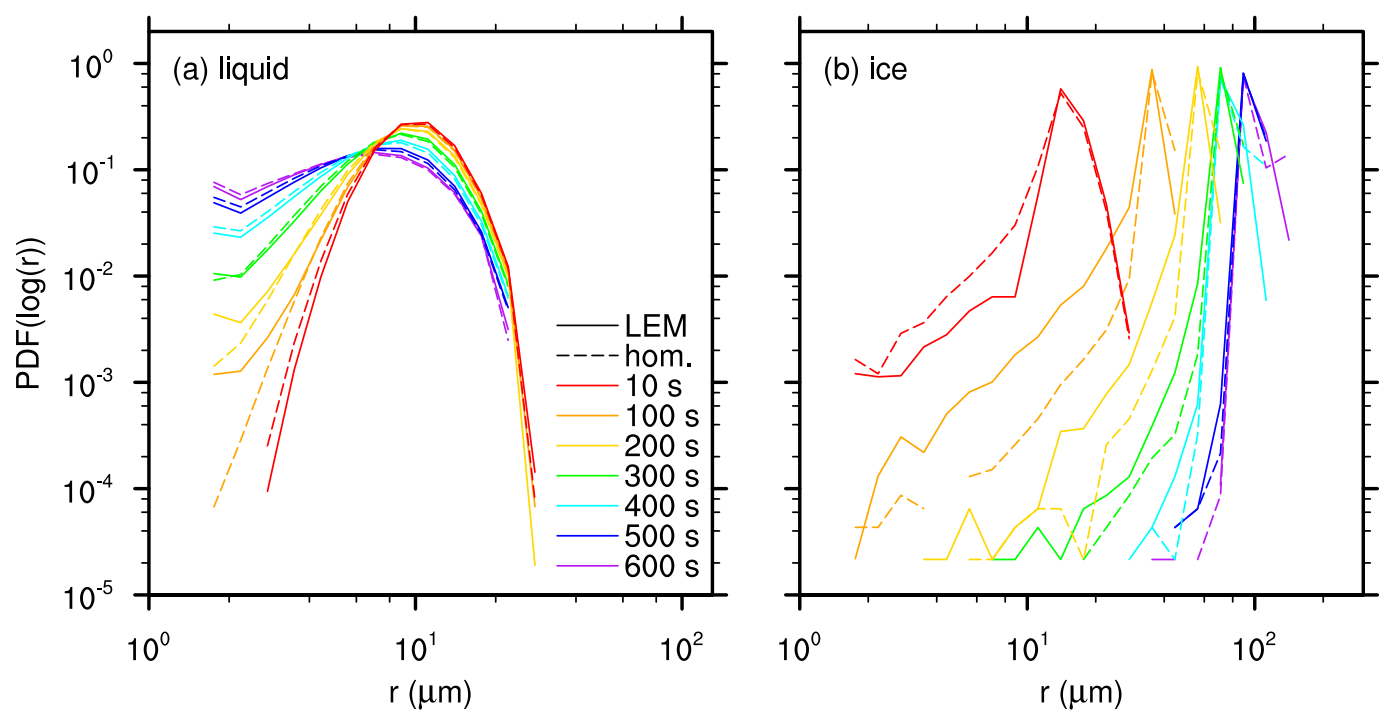

FIG. 3. Probability distribution functions (PDFs) of (a) the liquid droplet radii and (b) the ice crystal radii from the homogeneous box simulation (dashed lines) and the LEM (continuous lines), using the base-case setup. The colors indicate the simulated time at which these PDFs have been calculated.

kinetically limited, that is, restricted by the rate with which water molecules transit from the gas into the ice phase only, and not by the available ice supersaturation. Therefore, the ice water mixing ratio is determined by the number of ice crystals (e.g., Ervens et al. 2011; Yang et al. 2013). For $N_{\text {IN }}=1000 \mathrm{~L}^{-1}$, however, the ice supersaturations are depleted so quickly that there is no net flow of water molecules into the ice phase after $250 \mathrm{~s}$, which halts any further increase in ice water. Changes in the liquid water mixing ratio (Fig. 4b) are caused by evaporation following the initial mixing of the cloud with the entrained air $(t<150 \mathrm{~s})$ as well as the subsequent WBF process, resulting in a complete depletion of all liquid water after $250 \mathrm{~s}$ for $N_{\mathrm{IN}}=1000 \mathrm{~L}^{-1}$, and at commensurately later times for lower ice crystal concentrations (e.g., Korolev and Field 2008). This general behavior in $q_{i}$ and $q_{l}$ is qualitatively similar for the LEM and homogeneous simulations. However, quantitative differences can be discerned.

The effect of the LEM during the initial mixing of the cloud and the entrained segment is well understood. The ability of the LEM to spatially conserve the entrained air restricts evaporation to a small fraction of liquid droplets in the direct vicinity of the entrained air, while the uniform water subsaturation in the homogeneous simulation evaporates all liquid droplets in the entire model domain simultaneously at a commensurately faster time scale (e.g., Krueger 1993). This effect of the LEM is also indicated by Fig. 4d, showing the LEM liquid water mixing ratio divided by the corresponding quantity from the homogeneous simulation, $q_{l} / q_{l, \text { hom. }}$. And indeed, during the initial mixing, $q_{l} / q_{l, \text { hom. }}$ increases by $5 \%$. This surplus vanishes within $150 \mathrm{~s}$ as a result of the ongoing mixing, homogenizing the model domain for all $N_{\text {IN }}<$ $100 \mathrm{~L}^{-1}$. Similarly, the LEM ice water mixing ratio divided by the corresponding quantity from the homogeneous simulations, $q_{i} / q_{i \text { hom. }}$, also increases by about $2 \%$ for $t<20 \mathrm{~s}$ (Fig. 4c). This behavior can also be attributed to the spatial conservation of the cloud and the entrained segment in the LEM, providing preferential conditions for ice crystal growth inside the cloud.

The following changes, that is, the increase of $q_{l} / q_{l, \text { hom. }}$ for $N_{\mathrm{IN}} \geq 100 \mathrm{~L}^{-1}$ after $150 \mathrm{~s}$ and the decrease of $q_{i} / q_{i, \mathrm{hom}}$ to $-11 \%$ at about $t=80 \mathrm{~s}$, are caused by mixed-phase inhomogeneous processes that will be identified below.

\section{b. Identifying mixed-phase inhomogeneous processes}

The reason for the rapid decrease of $q_{i} / q_{i, \text { hom. }}$ to $-11 \%$ at about $t=80 \mathrm{~s}$ is a lower nucleation rate in the LEM simulations (Fig. 4e). While in the homogeneous case all entrained IN nucleate after $\tau_{\text {nuc }} \approx 100 \mathrm{~s}$, which we will assume as the nucleation time scale of all entrained IN in the following, the nucleation of all entrained IN is delayed by additional $50 \mathrm{~s}$ in the LEM simulations. The reason for this is the spatially resolved entrained segment that, because of its lower saturation ratio, is less favorable for deposition nucleation than the average-higher-saturation ratio in the homogeneous simulations. Accordingly, the temporarily lower number of ice crystals in the LEM simulation results in an overall slower increase of $q_{i}$ in the LEM simulation. As seen in Fig. 2, the ongoing mixing of the entrained air with the 

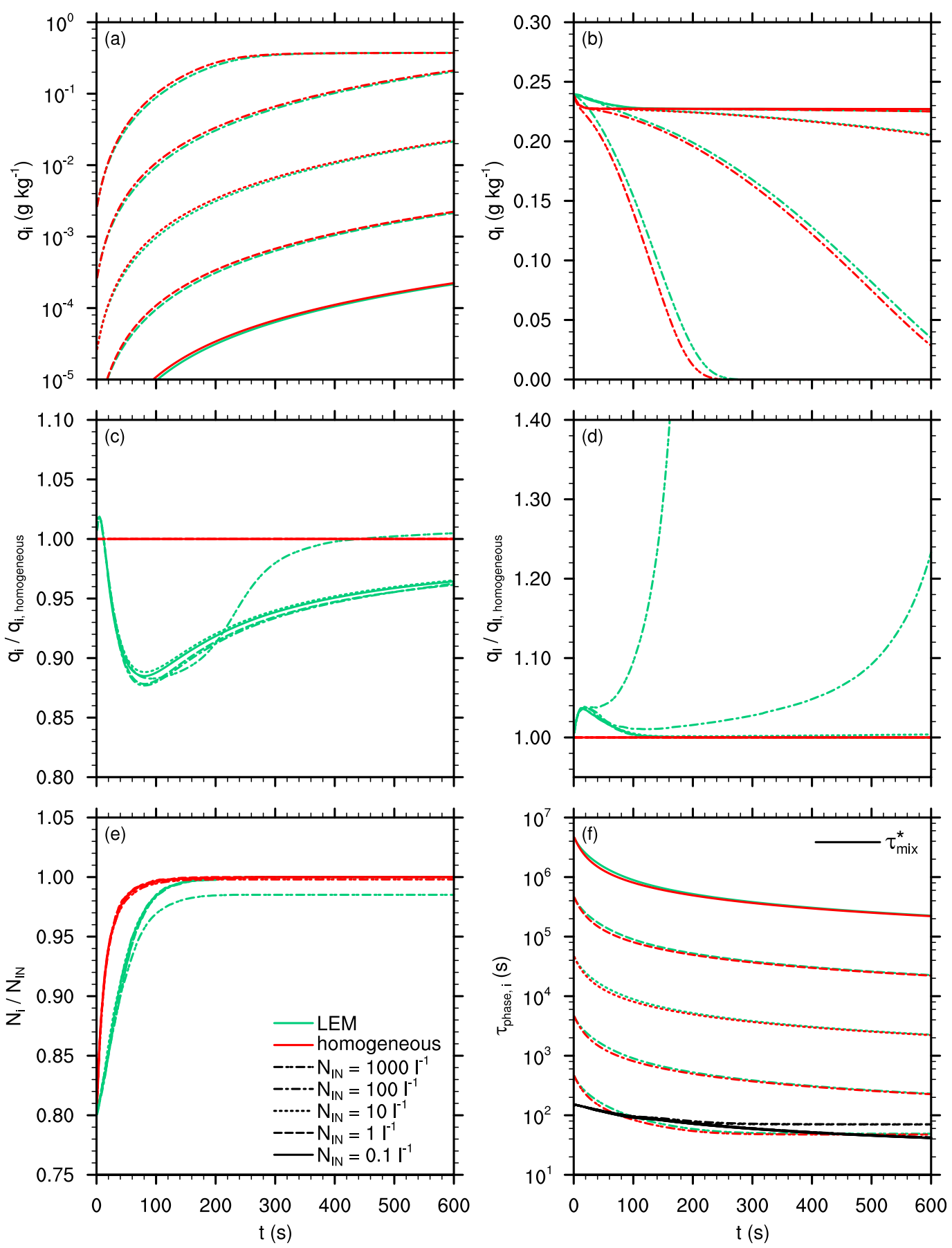

FIG. 4. Time series of (a) the ice water mixing ratio, (b) liquid water mixing ratio, (c) ratio of ice water mixing ratio in the LEM to that from the homogeneous simulation, (d) ratio of liquid water mixing ratio in the LEM to that from the homogeneous simulation, (e) fraction of nucleated IN, and (f) ice-phase relaxation time scale for simulations based on the base case with varied initial IN concentrations (line patterns). Results from the LEM and the homogeneous simulations are presented in green and red lines, respectively. The black lines in (f) show the generalized mixing time scale of the LEM. 
cloud transports these IN into the cloud where they nucleate after some time, which is also the reason for the broader tail toward smaller ice crystal equivalent radii evident in Fig. 3b. After these IN are nucleated, the ice water mixing ratios of the LEM simulations approach the values of the homogeneous simulations. For all LEM simulations with $N_{\text {IN }} \leq 100 \mathrm{~L}^{-1}$, this happens at the same time scale determined by the kinetically limited ice crystal growth. For $N_{\text {IN }}=1000 \mathrm{~L}^{-1}$, however, this adjustment is somewhat faster since the growth is limited by the available ice supersaturation, which is approximately the same in the LEM and the homogeneous simulations. All in all, we see a decreased ice water mixing ratio in the LEM simulations as a result of the delayed nucleation of entrained IN. This is a typical inhomogeneous reaction since the generalized mixing time scale $\tau_{\text {mix }}^{*} \approx 159 \mathrm{~s}$ is initially longer than the nucleation time scale $\tau_{\text {nuc }} \approx 100 \mathrm{~s}$, resulting in a Damköhler number larger than 1. In the following, we will call this process spatially inhomogeneous nucleation.

The increase of $q_{l} / q_{l, \text { hom. }}$ for $N_{\text {IN }} \geq 100 \mathrm{~L}^{-1}$ and $t>150 \mathrm{~s}$ is associated with inhomogeneities in the hydrometeor fields (Fig. 4d). These inhomogeneities are regions inside the cloud devoid of ice crystals but filled with liquid droplets or, vice versa, regions devoid of liquid droplets but filled with ice crystals. In the prior case, the liquid droplets of the considered region do not evaporate since they do not experience the water subsaturations produced by ice crystals outside the region, while in the latter case, water subsaturations produced by the ice crystals in the considered region are too localized to affect droplet evaporation outside the region. In both cases, however, WBF is only decelerated if (i) the flux of water vapor from or to the localized heterogeneity is sufficiently reduced by slow turbulent mixing, and (ii) the ice crystal growth is sufficiently fast so as to be influenced by this lack of water vapor. Accordingly, an ice phase relaxation time scale shorter than the mixing time scale is required to reduce the WBF process, that is, a Damköhler number larger than 1 .

This is analyzed in Fig. 4f, which shows the ice phase relaxation time scale (colored lines) and the generalized mixing time scale (only relevant for the LEM, black lines). Both time scales decrease with time, primarily due to ice crystal growth, which accelerates phase changes due to a larger surface area but also the mixing due to faster sedimentation as a result of the increasing ice crystal mass. The decrease in the generalized mixing time scale is, however, relatively small because of the generally small sedimentation velocity of ice crystals (e.g., Mitchell 1996), indicating that turbulence is the main driver for mixing in the conducted simulations.

Overall, the increase of $q_{l} / q_{l, \text { hom. }}$ for $t>150 \mathrm{~s}$ coincides with the cases $N_{\mathrm{IN}} \geq 100 \mathrm{~L}^{-1}$ in which $\tau_{\text {mix }}^{*} \geq \tau_{\text {phase }, i}$, that is, cases with a Damköhler number larger than 1 that predicts an inhomogeneous reaction. For smaller $N_{\mathrm{IN}}$, $\tau_{\text {mix }}^{*} \ll \tau_{\text {phase }, i}$, which implies a homogeneous reaction in which mixing is sufficiently fast to homogenize the hydrometeor and thermodynamic fields, eliminating the potential influence of any small-scale structures.

On the whole, this shows that the WBF process can be reduced by inhomogeneous mixing, but only if the number of ice crystals is very high (as occurring in the simulations with $N_{\text {IN }} \geq 100 \mathrm{~L}^{-1}$ ). Consequently, this process only affects an already rapid WBF, naturally limiting the temporal delay as well as the amount of liquid water that is preserved in comparison to the homogeneous simulations (Fig. 4b). Additionally, a wide range of $N_{\text {IN }}$ conditions is not affected by this process, supporting the commonly made assumption of homogeneity on the unresolved scales of many models with coarser resolutions.

Note, however, that the previously discussed spatially inhomogeneous nucleation reduces the number and size of ice crystals temporarily and therefore increases the ice phase relaxation time scale, with commensurate effects on the potentially inhomogeneous character of the WBF process. To analyze the interaction of these processes further, we conducted a simulation without entrained IN, which prevents spatially inhomogeneous nucleation by design (Fig. 5). Due to the generally smaller number of IN (Fig. 5e), the simulations without entrained IN slightly delay the increase of ice water (Fig. 5a) and the depletion of liquid water by WBF (Fig. 5b) as expected. In general, however, the abovedescribed effects of the inhomogeneous mixing of the liquid droplets following the entrainment event is similar, that is, slightly higher ice and liquid water mixing ratios for $t<100-150$ s (Figs. 5c,d). Also, the inhomogeneous WBF reduction occurs (Fig. 5d), but only for $N_{\text {IN }}=$ $1000 \mathrm{~L}^{-1}$, that is, at a higher ice crystal concentration than for Fig. 4. Again, the reason is the overall reduced IN concentration after entrainment, requiring a higher incloud ice crystal concentration to decrease the ice phase relaxation time scale below the mixing time scale (Fig. 5f). This reemphasizes that the inhomogeneous reduction in WBF requires high ice crystal concentrations to be effective, and a reduction in the ice crystal concentration due to entrainment makes the WBF process more homogeneous.

\section{c. Effects of entrainment and mixing}

Now, the effects of entrainment and mixing on the WBF process will be investigated, focusing on 

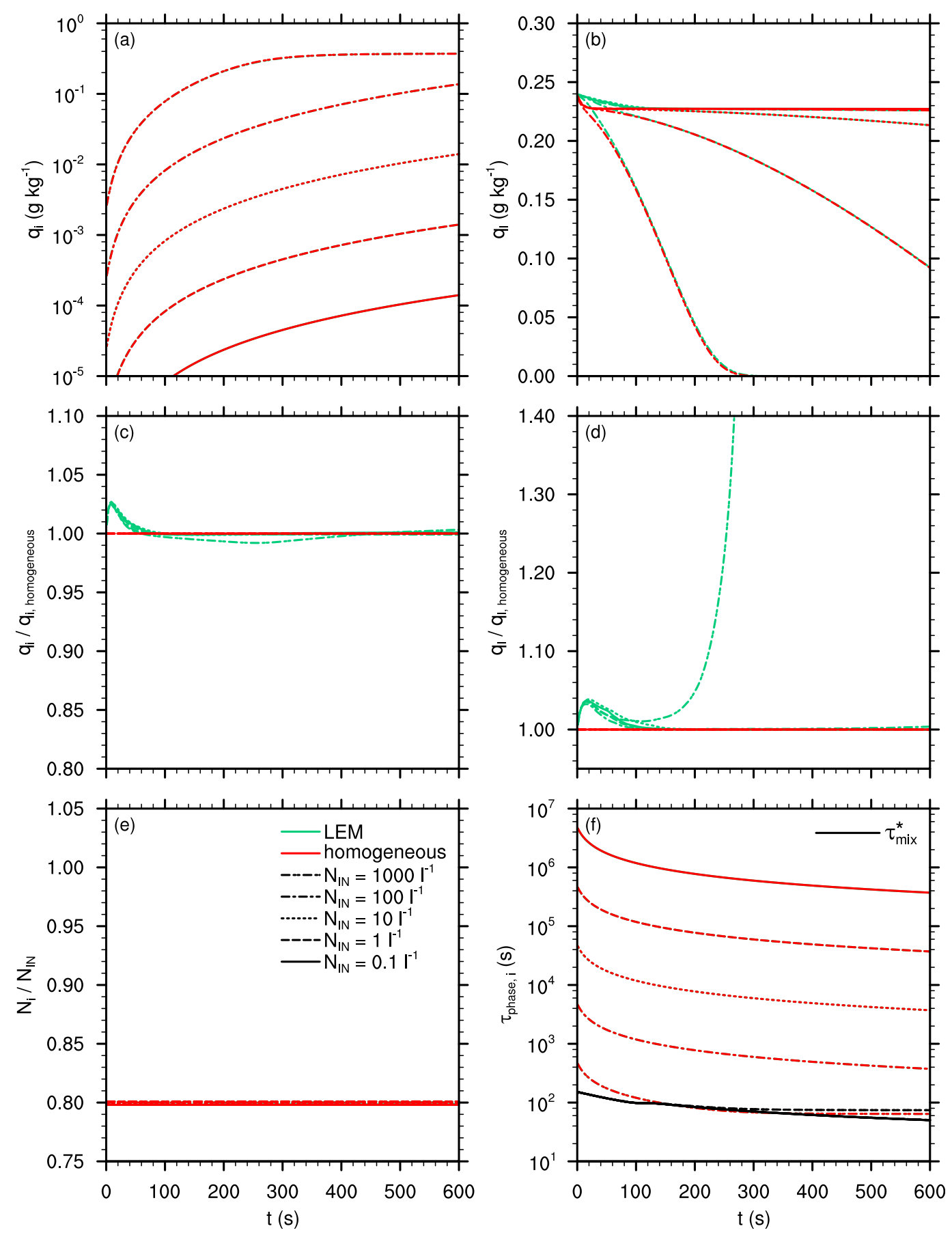

FIG. 5. As in Fig. 4, but based on simulations without entrained IN.

the impact of the kinetic energy dissipation rate $\epsilon$, which is a measure of the degree of turbulence, and the fraction of entrained air $f_{\text {entr }}$ (Figs. 6,7).

The degree of turbulence does not affect the ice or liquid water mixing ratio of the homogeneous simulations for which instantaneous mixing is assumed (Figs. 6a,b). In the LEM simulations, however, weaker turbulence decelerates the increase in ice water mixing ratio, while it maintains higher liquid water mixing ratios, indicating a more inhomogeneous mixing of the entrained segment with the cloud and a stronger inhomogeneous reduction of WBF.

For a larger fraction of entrained air (Fig. 7), the liquid water mixing ratio decreases as the fraction of entrained 

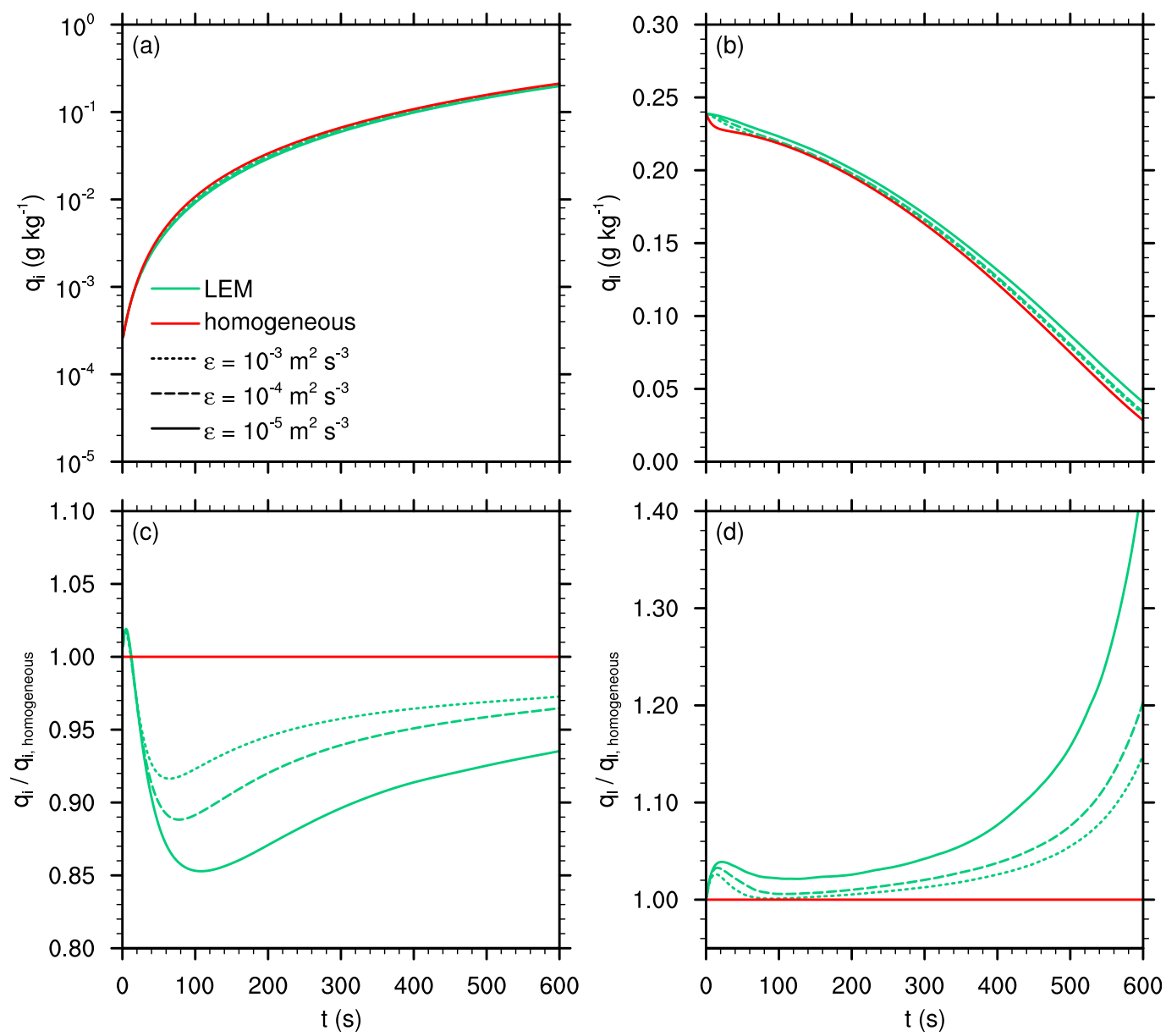

FIG. 6. Time series of (a) the ice water mixing ratio, (b) liquid water mixing ratio, (c) ratio of ice water mixing ratio in the LEM to that from the homogeneous simulation, and (d) ratio of liquid water mixing ratio in the LEM to that from the homogeneous simulation for simulations based on the base case with varied kinetic energy dissipation rates (line patterns). Results from the LEM and the homogeneous simulations are presented in green and red lines, respectively.

air increases due to stronger dilution and hence evaporation of liquid droplets (Fig. 7b). A similar reduction of the ice water mixing ratio after the entrainment event is only visible in the first $100 \mathrm{~s}$, and is comparably small (Fig. 7a). Afterward, however, the ice water mixing ratio actually increases for a larger entrainment fraction since the dilution of the cloud provides more water vapor for ice crystal growth due to the commensurately increased evaporation of the liquid droplets following the entrainment event. Accordingly, entrainment boosts WBF by accelerating the evaporation of liquid droplets.

Differences between the LEM and the homogeneous simulations indicate, however, that the aforementioned inhomogeneous processes counteract the accelerated WBF in entraining clouds. Weaker turbulence and a larger fraction of entrained air keep the entrained IN in an environment less favorable for deposition nucleation and hence decelerate the increase in ice water (Figs. 6c, 7c). Similarly, weaker turbulence and a larger fraction of entrained air delay the depletion of the liquid water due to WBF (Figs. 6d, 7d). Both effects are in agreement with the definition of the turbulent mixing time scale (2), where a smaller $\epsilon$ or a larger $l=L f_{\text {entr }}$ indicate a slower mixing process that allows inhomogeneities to exist for a longer time, reducing the transport of water vapor from the liquid to the ice phase as discussed above.

This effect is further analyzed in Fig. 8, in which the average distance of an ice crystal to the closest liquid droplet within the LEM domain is displayed for the analyzed kinetic energy dissipation rates (Fig. 8a) and fractions of entrained air (Fig. 8b). While the distance is initially small, it increases quickly after the beginning 

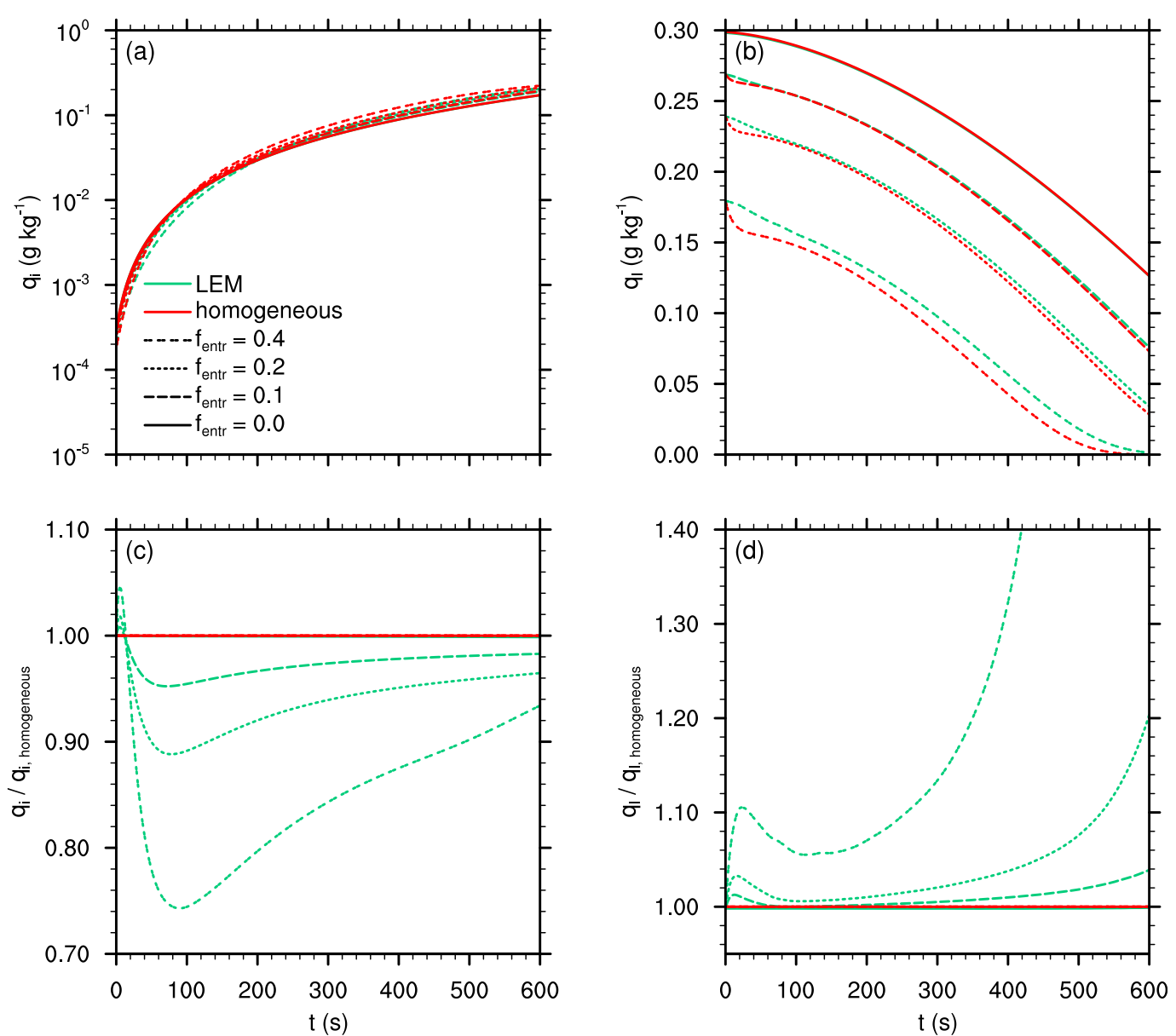

FIG. 7. As in Fig. 6, but with varied entrainment fractions (line patterns).

of the simulation when inhomogeneities caused by the entrainment event begin to affect the distribution of ice and liquid droplets $(t<20 \mathrm{~s})$; for example, ice crystals fall into the entrained air or chunks of entrained air are mixed into the cloud. From their maximum values, these distances decrease again $(t<200 \mathrm{~s})$, indicating the mixing and eventual homogenization of the entrained air within the cloud, which is scaled by $\epsilon$ and $f_{\text {entr }}$ as expected. However, the average distance of an ice crystal to the closest liquid droplet starts to increase again toward the end of the simulation. Since the entrainment and mixing process is largely finished, these voids are most likely created by the complete evaporation of individual liquid droplets in the vicinity of ice crystals; that is, WBF itself causes inhomogeneities. This process counteracts WBF since the ice crystals located in these voids are unable to grow any further due to a lack of water vapor. Note that this process is even occurring for $f_{\text {entr }}=0$, indicating that entrainment is not necessary for this effect. Nonetheless, it is accelerated by a generally larger distance between the hydrometeors as it is caused by a larger fraction of entrained air (Fig. 8b).
All in all, this section shows that entrainment generally accelerates WBF by contributing to the evaporation of droplets, which increases the available water vapor for ice crystal growth. Small-scale inhomogeneities in the ice crystal or liquid droplet fields are found to counteract this effect by reducing the flux of water vapor from the liquid to the ice phase. These inhomogeneities are shown to be created by the entrainment process or by the WBF process on its own, causing the complete evaporation of liquid droplets in the vicinity of ice crystals.

\section{d. Vertical motion}

While entrainment tends to accelerate WBF, small-scale inhomogeneities tend to decelerate it. Accordingly, these effects are similar to the effects of up- and downdrafts on WBF, where positive vertical velocities decelerate WBF and negative accelerate it (Korolev and Field 2008; Ervens et al. 2011). To compare entrainment, mixing, and vertical velocities, Fig. 9 shows the impact of mean up- and downdrafts $(\bar{w}= \pm 10$ and 

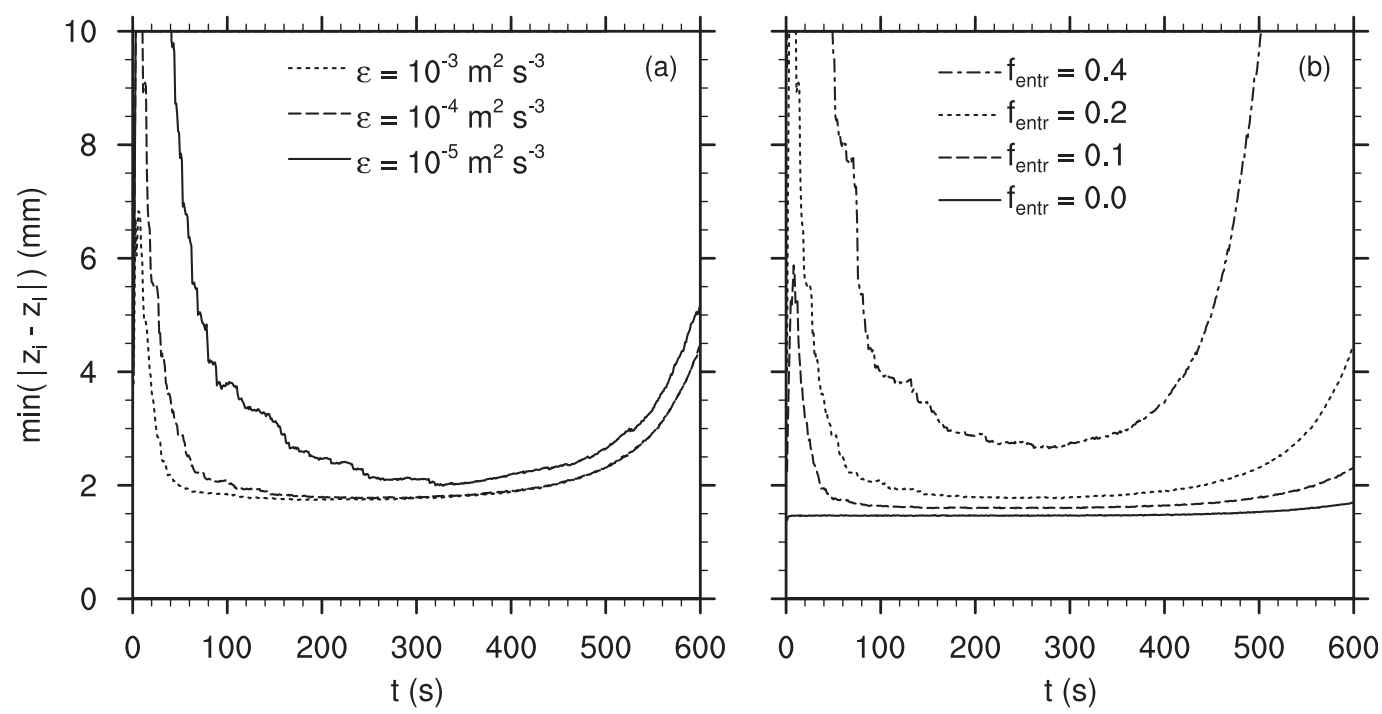

FIG. 8. Time series of the average distance between an ice crystal and the next liquid droplet, depending on (a) kinetic energy dissipation rate and (b) entrainment fraction (line patterns).

$\pm 2 \mathrm{~cm} \mathrm{~s}^{-1}$ ) on the development of the investigated base state cloud parcel. As expected, a positive vertical velocity delays the depletion of liquid water, while a negative vertical velocity increases it (Fig. 9b). However, there is no significant effect on the ice water (Fig. 9a). The reason for this is the much larger integral surface of the liquid droplets, resulting in a much shorter phase relaxation time scale of this hydrometeor species, allowing them to react much faster to the additional cooling or heating. This behavior, however, is only possible for the moderate vertical velocities tested here with commensurately low cooling or heating rates, while higher vertical velocities are expected to affect the ice water more significantly (e.g., Korolev and Field 2008; Ervens et al. 2011).

Overall, the explicit consideration of inhomogeneities by the LEM affects the simulations with a vertical velocity as previously discussed. The additional cooling or heating due to the vertical velocities affects the processes only marginally. It is, however, interesting to note that the inhomogeneous reduction of WBF due to the explicit treatment by the LEM is approximately similar to the effect of a mean updraft of $2 \mathrm{~cm} \mathrm{~s}^{-1}$ in the homogeneous simulation (Fig. 9b).

\section{e. Synthesis}

The previous analysis shows clearly that spatially inhomogeneous nucleation reduces the ice water mixing ratio in the LEM compared to homogeneous simulations, while the inhomogeneously reduced WBF increases the liquid water mixing ratio in the LEM compared to homogeneous simulations. To highlight this general behavior, Fig. 10 compiles 1$) \min \left(q_{i} / q_{i, \text { hom. }}\right)$, indicating the effect of spatially inhomogeneous nucleation, and 2) $\max \left(q_{l} / q_{l, \text { hom. }}\right)$, demonstrating the impact of inhomogeneous reduction of WBF, in a phase space defined by the generalized mixing time scale on the ordinate and the ice phase relaxation time scale on the abscissa. Both time scales are diagnosed when the respective extremum occurs. The lines $\mathrm{Da}_{\text {nuc }}=\tau_{\mathrm{mix}}^{*} / \tau_{\text {nuc }}=1$, with an assumed nucleation time scale $\tau_{\text {nuc }} \approx 100 \mathrm{~s}$, and $\mathrm{Da}_{\text {phase }, i}=\tau_{\text {mix }}^{*} / \tau_{\text {phase }, i}=1$ indicate the transition between inhomogeneous and homogeneous reactions in the phase space. The reader is referred to the introduction for the definition of these quantities. Only data from the previous deposition-nucleation simulations with $\bar{w}=0 \mathrm{~cm} \mathrm{~s}^{-1}$ are presented.

All in all, one can see that the change in $\min \left(q_{i} / q_{i \text { hom. }}\right)$ tends to show a reduction of the ice water mixing ratio due to spatially inhomogeneous nucleation if $\mathrm{Da}_{\text {nuc }} \geq 1$. Similarly, $\max \left(q_{l} / q_{l, \text { hom. }}\right)$ shows a distinct increase when $\mathrm{Da}_{\text {phase }, i}$ is close to 1 , indicating inhomogeneously reduced WBF. Accordingly, these plots show that if the requirements on the time scales for mixing, ice phase relaxation, and nucleation are met, the mixedphase inhomogeneous processes identified in this study, namely, spatially inhomogeneous nucleation and the inhomogeneously reduced WBF, may have significant impacts on the ice and liquid water contents of the affected clouds.

\section{Discussion and conclusions}

The leading question of this study has been: How do entrainment and mixing affect mixed-phase clouds? 

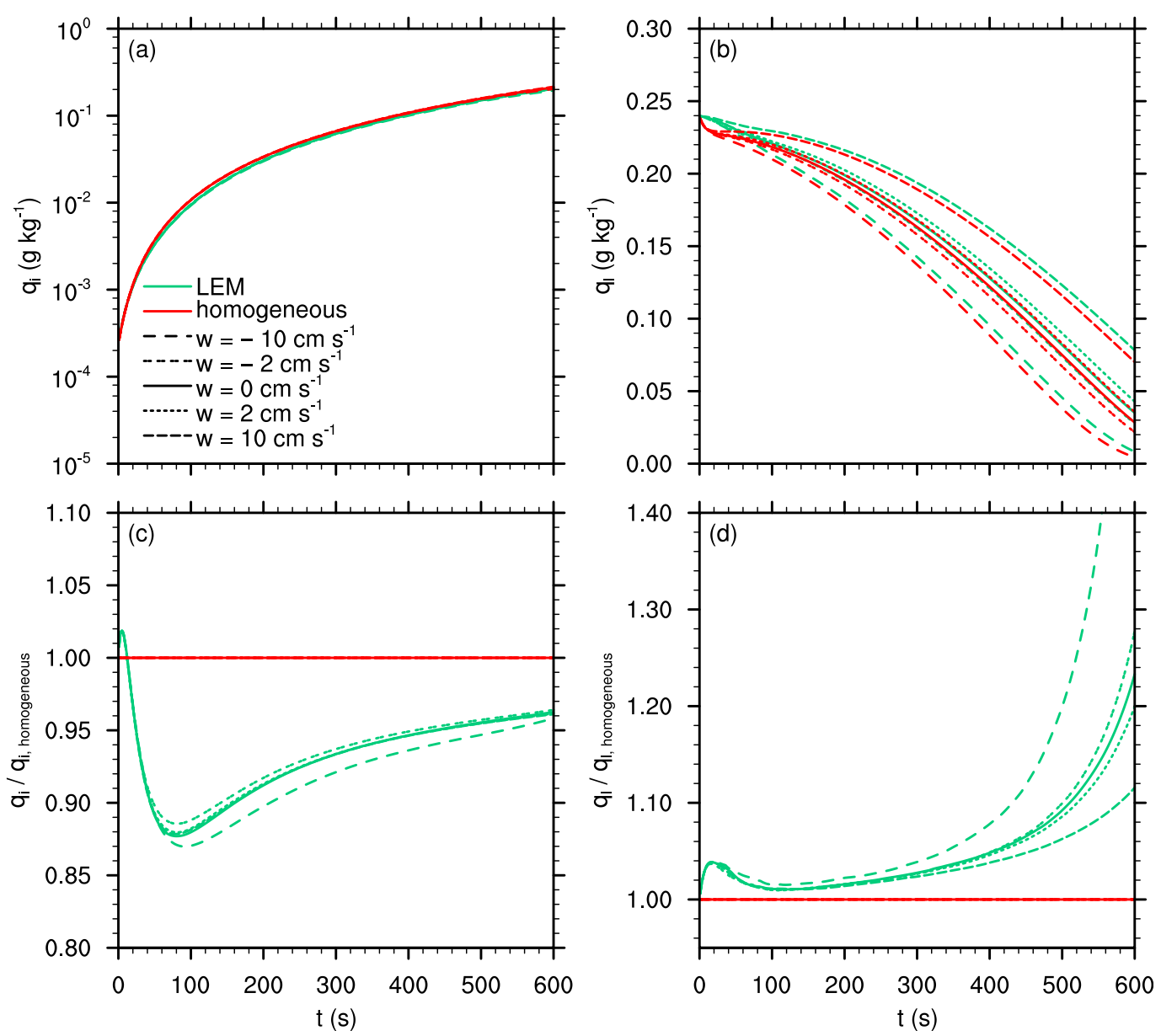

FIG. 9. Time series of (a) the ice water mixing ratio, (b) liquid water mixing ratio, (c) ratio of ice water mixing ratio in the LEM to that from the homogeneous simulation, and (d) ratio of liquid water mixing ratio in the LEM to that from the homogeneous simulation for simulations based on the base case with varied vertical velocity (line patterns). Results from the LEM and the homogeneous simulations are presented in green and red lines, respectively.

This question has been approached using a modeling framework that allows representation of all relevant spatial scales imposed by turbulent entrainment and mixing explicitly, that is, from a resolution of a couple of millimeters to a hectometer model domain, including the required detail in the representation of individual ice crystals and cloud droplets. Since the typical resolution of today's large-scale models is usually coarser than tens to hundreds of meters, this study also reveals details that are usually neglected in these models that assume homogeneity on unresolved scales.

While this study shows that an entrainment event generally accelerates the glaciation of a cloud through the Wegener-Bergeron-Findeisen (WBF) process by contributing to the evaporation of liquid droplets, smallscale processes associated with entrainment and mixing decelerate WBF. These processes are identified as spatially inhomogeneous nucleation and inhomogeneous reduction of WBF.

Spatially inhomogeneous nucleation requires that the nucleation time scale, typically between 10 and $100 \mathrm{~s}$ in mixed-phase clouds (e.g., Ervens and Feingold 2012), is shorter than the mixing time scale necessary to homogenize the entrained air with the cloud. If this requirement is fulfilled, the nucleation of the entrained IN is delayed due to the typically less favorable conditions for nucleation in the entrained air. Accordingly, the explicit representation of the entrainment and mixing process delays the nucleation of entrained IN compared to a homogeneous simulation, in which the mixing is forced to be instantaneous as usually done on the unresolved scales of a large-scale model. Due to the temporary smaller number of ice crystals, the increase of ice water in the 

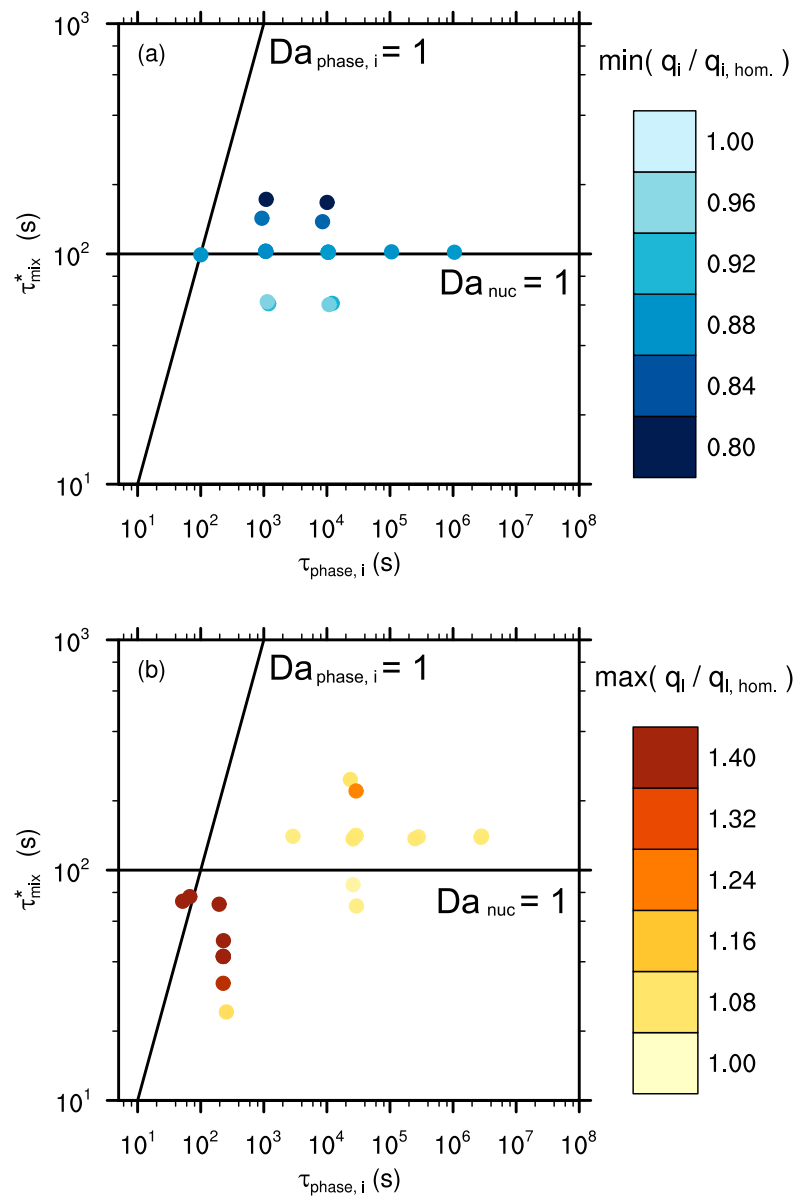

FIG. 10. Phase-space representation of (a) $\min \left(q_{i} / q_{i, \text { hom. }}\right)$ and (b) $\max \left(q_{l} / q_{l, \text { hom. }}\right)$ for various ensemble-averaged, depositionnucleation simulations. The phase space is defined by the ice-phase relaxation time scale and the generalized mixing time scale. The lines $\mathrm{Da}_{\text {nuc }}=1$ and $\mathrm{Da}_{\text {phase }, i}=1$ indicate the transition between inhomogeneous and homogeneous effects of nucleation and WBF reduction, respectively.

affected cloud is reduced. In this study, spatially inhomogeneous nucleation has only been analyzed for clouds that entrain IN that nucleate by deposition. Clouds entraining immersion-freezing IN, however, might also be affected but require a significant updraft to grow the entrained aerosol particles to cloud droplets before freezing. And indeed, the activation and subsequent growth of aerosol particles entrained above the cloud base may occur in cumuliform clouds (e.g., Hoffmann et al. 2015), while this process can be excluded for stratiform clouds in which entrained air moves downward, preventing activation and further droplet growth (e.g., Hoffmann and Feingold 2019). However, it is important to note that there is still large uncertainty in the modeling of ice nucleation, especially regarding the associated time scales, including completely time-independent formulations, with commensurate implications for spatially inhomogeneous nucleation (e.g., Kanji et al. 2017).

Inhomogeneous reduction of WBF occurs if turbulence mixing is so slow that voids in the ice crystal or liquid droplet field are distinct from the surrounding air, reducing the transfer of water vapor from the liquid to the ice phase, which delays the glaciation of the cloud. While we show that ice crystal voids are produced by the entrainment of environmental air, voids in the liquid droplet field can be produced by a very efficient WBF on its own due to the complete evaporation of liquid droplets in the vicinity of an ice crystal. To initiate the inhomogeneous reduction of WBF, the mixing time scale needs to be longer than the ice phase relaxation time scale that-because of the natural paucity of ice crystals - often exceeds several hours. Therefore, only clouds with correspondingly high ice crystal concentrations exceeding 100 to $1000 \mathrm{~L}^{-1}$, for which the ice phase relaxation time scale is on the order of hundreds of seconds, are affected by this process. Thus, typical Arctic mixed-phase clouds might only be marginally affected by this process since they exhibit ice crystal concentrations between 0.1 and $100 \mathrm{~L}^{-1}$ (Morrison et al. 2012). Deeper mixed-phase clouds with active secondary ice production exhibit ice crystal concentrations of up to $10^{4} \mathrm{~L}^{-1}$, making these clouds highly susceptible to this process. On the other hand, WBF at these high ice crystal concentrations is very efficient, which significantly restricts the time scale on which the inhomogeneous reduction of WBF might affect a cloud.

All in all, this study demonstrates the existence of inhomogeneous processes in mixed-phase clouds on the scale of meters and larger, that is, on scales that are typically unresolved in large-scale models and hence assumed to be homogeneous. We have shown that the importance of these processes for the development of a cloud depends strongly on the cloud microphysical composition, and hence the assumption of homogeneity might be justified in many cases. This begs the question: When do we need to consider, and represent these inhomogeneous processes in our cloud models? Due to its idealized character, the current study can only provide an initial process-level understanding. To assess the occurrence and importance of these processes more broadly, simulation of cloud fields are mandatory. Large-eddy simulation (LES) models have proved to be a useful framework for capturing dynamical-microphysical interactions in evolving cloud fields, but the necessary small-scale details required to represent inhomogeneities in the entrainment-mixing process are typically inaccessible due to computational constraints on model resolution. In a follow-up study, we plan to apply the L3 model, an LES with Lagrangian cloud microphysics and 
the above-introduced LEM as a subgrid-scale model (Hoffmann et al. 2019; Hoffmann and Feingold 2019). By bridging the gap between the small-scale detail required to represent inhomogeneous processes, and the capability to represent cloud fields, L3 will enable a much better assessment of the importance of the mixedphase inhomogeneous processes revealed in this study, in dynamically coupled, evolving mixed-phase cloud simulations. These L3 results may not only constitute the basis for potential parameterization development of the above-described inhomogeneous mixed-phase processes to be used in larger-scale models, but they may also increase our general understanding of mixed-phase clouds in the climate system. Given the strong sensitivity of these clouds to process representation (e.g., Solomon et al. 2015), and the potential for bifurcation between cloudy and cloud-free states (Morrison et al. 2012), this seems like a promising line of inquiry.

Acknowledgments. Fabian Hoffmann thanks Graham Feingold and Franziska Glassmeier for constructive discussions, as well as Steve Krueger and two anonymous reviewers for their comments on the manuscript. This research was performed while Fabian Hoffmann held a Visiting Fellowship of the Cooperative Institute for Research in Environmental Sciences (CIRES) at the University of Colorado Boulder and the NOAA Earth System Research Laboratory.

\section{APPENDIX}

\section{The Glaciation and the Phase Relaxation Time Scale}

As shown by Korolev and Mazin (2003), the time to convert all liquid water to ice by the WBF process, the glaciation time scale $\tau_{\mathrm{gl}}$, can be derived from the diffusional growth equation for the mass of an ice crystal,

$$
\frac{d m_{i}}{d t} \approx 4 \pi D_{v} c r_{i}\left(\rho_{v}-\rho_{s, i}\right),
$$

where $\rho_{v}$ is the vapor density and $\rho_{s, i}$ the ice-saturation vapor density. Typical for mixed-phase clouds, it is assumed that the vapor density is at liquid water saturation, $\rho_{v}=\rho_{s, l}$. It is further assumed that initially no ice is present, that is, the ice water mixing ratio after glaciation (at $t=\tau_{\mathrm{gl}}$ ) equals the initial liquid water mixing ratio (at $t=t_{0}=0$ ),

$$
q_{i}\left(\tau_{\mathrm{gl}}\right)=q_{l, 0}
$$

Accordingly, the mass of an ice crystal at the beginning and the end of WBF is $m_{i}\left(t_{0}\right)=0$ and $m_{i}\left(\tau_{\mathrm{gl}}\right)=$ $q_{l}\left(t_{0}\right) \rho_{a} / N_{i}$, respectively. Integration of (A1) yields

$$
\tau_{\mathrm{gl}} \approx \frac{3}{2} \frac{1}{4 \pi D_{v} c\left(\rho_{s, l}-\rho_{s, i}\right)}\left(\frac{4}{3} \pi \rho_{i}\right)^{1 / 3}\left(\frac{q_{l, 0} \rho_{a}}{N_{i}}\right)^{2 / 3},
$$

similar to Korolev and Mazin (2003).

By introducing the phase relaxation time scale for ice crystals,

$$
\tau_{\text {phase }, i} \approx\left(4 \pi D_{v} N_{i} c r_{i}\right)^{-1},
$$

we can rewrite (A3) as

$$
\begin{aligned}
\tau_{\mathrm{gl}} & \approx \frac{3}{2} \tau_{\text {phase }, i} \frac{r_{i} N_{i}}{\rho_{s, l}-\rho_{s, i}}\left(\frac{4}{3} \pi \rho_{i}\right)^{1 / 3}\left(\frac{q_{l, 0} \rho_{a}}{N_{i}}\right)^{2 / 3} \\
& =\frac{3}{2} \tau_{\text {phase }, i} \frac{q_{l, 0}}{q_{s, l}-q_{s, i}},
\end{aligned}
$$

using the fact that $r_{i}=\left(4 \pi \rho_{i} / 3\right)^{-1 / 3}\left(q_{l, 0} \rho_{a} / N_{i}\right)^{1 / 3}$ at $t=\tau_{\mathrm{gl}}$. Here, $q_{s, l}$ and $q_{s, i}$ are the liquid-water and ice saturation vapor mixing ratios, respectively.

By assuming typical mixed-phase cloud parameters, one sees that $\tau_{\mathrm{gl}}$ is mainly determined by $\tau_{\text {phase }, i}$, which varies by several orders of magnitude, while $q_{l, 0}$ and $q_{s, l}-q_{s, i}$ are usually of the same order of magnitude, mitigating their individual influence.

\section{REFERENCES}

Baker, M., and J. Latham, 1979: The evolution of droplet spectra and the rate of production of embryonic raindrops in small cumulus clouds. J. Atmos. Sci., 36, 1612-1615, https://doi.org/ 10.1175/1520-0469(1979)036<1612:TEODSA $>2.0$. CO; 2 .

Beard, K. V., 1976: Terminal velocity and shape of cloud and precipitation drops aloft. J. Atmos. Sci., 33, 851-864, https://doi.org/ 10.1175/1520-0469(1976)033<0851:TVASOC $>2.0$. CO 2 .

Bergeron, T., 1935: On the physics of clouds and precipitation. Proc. Fifth Assembly, Lisbon, Portugal, International Union of Geodesy and Geophysics, 156-180.

Bodenschatz, E., S. P. Malinowski, R. A. Shaw, and F. Stratmann, 2010: Can we understand clouds without turbulence? Science, 327, 970-971, https://doi.org/10.1126/science.1185138.

Chen, J.-P., and D. Lamb, 1994: The theoretical basis for the parameterization of ice crystal habits: Growth by vapor deposition. J. Atmos. Sci., 51, 1206-1222, https://doi.org/10.1175/ 1520-0469(1994)051<1206:TTBFTP>2.0.CO;2.

_- A. Hazra, and Z. Levin, 2008: Parameterizing ice nucleation rates using contact angle and activation energy derived from laboratory data. Atmos. Chem. Phys., 8, 7431-7449, https:// doi.org/10.5194/acp-8-7431-2008.

Chylek, P., and C. Borel, 2004: Mixed phase cloud water/ice structure from high spatial resolution satellite data. Geophys. Res. Lett., 31, L14104, https://doi.org/10.1029/2004GL020428.

Ervens, B., and G. Feingold, 2012: On the representation of immersion and condensation freezing in cloud models using different nucleation schemes. Atmos. Chem. Phys., 12, 58075826, https://doi.org/10.5194/acp-12-5807-2012.

, - - K. Sulia, and J. Harrington, 2011: The impact of microphysical parameters, ice nucleation mode, and habit growth on the 
ice/liquid partitioning in mixed-phase Arctic clouds. J. Geophys. Res., 116, D17205, https://doi.org/10.1029/2011JD015729.

Findeisen, W., 1938: Kolloid-meteorologische Vorgänge bei Neiderschlags-bildung. Meteor. Z., 55, 121-133.

Hoffmann, F., and G. Feingold, 2019: Entrainment and mixing in stratocumulus: Effects of a new explicit subgrid-scale scheme for large-eddy simulations with particle-based microphysics. J. Atmos. Sci., 76, 1955-1973, https://doi.org/10.1175/JAS-D18-0318.1.

- S. Raasch, and Y. Noh, 2015: Entrainment of aerosols and their activation in a shallow cumulus cloud studied with a coupled LCM-LES approach. Atmos. Res., 156, 43-57, https:// doi.org/10.1016/j.atmosres.2014.12.008.

— , Y. Noh, and S. Raasch, 2017: The route to raindrop formation in a shallow cumulus cloud simulated by a Lagrangian cloud model. J. Atmos. Sci., 74, 2125-2142, https://doi.org/10.1175/ JAS-D-16-0220.1.

— - T. Yamaguchi, and G. Feingold, 2019: Inhomogeneous mixing in Lagrangian cloud models: Effects on the production of precipitation embryos. J. Atmos. Sci., 76, 113-133, https:// doi.org/10.1175/JAS-D-18-0087.1.

Howell, W. E., 1949: The growth of cloud drops in uniformly cooled air. J. Meteor., 6, 134-149, https://doi.org/10.1175/1520-0469(1949) 006<0134:TGOCDI $>2.0$. CO;2.

Kanji, Z. A., L. A. Ladino, H. Wex, Y. Boose, M. Burkert-Kohn, D. J. Cziczo, and M. Krämer, 2017: Overview of ice nucleating particles. Ice Formation and Evolution in Clouds and Precipitation: Measurement and Modeling Challenges, Meteor. Monogr., No. 58, Amer. Meteor. Soc., https:// doi.org/10.1175/AMSMONOGRAPHS-D-16-0006.1.

Kerstein, A. R., 1988: A linear-eddy model of turbulent scalar transport and mixing. Combust. Sci. Technol., 60, 391-421, https://doi.org/10.1080/00102208808923995.

Korolev, A. V., and I. P. Mazin, 2003: Supersaturation of water vapor in clouds. J. Atmos. Sci., 60, 2957-2974, https://doi.org/ 10.1175/1520-0469(2003)060<2957:SOWVIC $>2.0$.CO;2.

- , and P. R. Field, 2008: The effect of dynamics on mixed-phase clouds: Theoretical considerations. J. Atmos. Sci., 65 66-86, https://doi.org/10.1175/2007JAS2355.1.

- and Coauthors, 2017: Mixed-phase clouds: Progress and challenges. Ice Formation and Evolution in Clouds and Precipitation: Measurement and Modeling Challenges, Meteor. Monogr., No. 58, Amer. Meteor. Soc., https://doi.org/10.1175/ AMSMONOGRAPHS-D-17-0001.1.

Krueger, S. K., 1993: Linear eddy modeling of entrainment and mixing in stratus clouds. J. Atmos. Sci., 50, 3078-3090, https://doi.org/10.1175/1520-0469(1993)050<3078:LEMOEA > 2.0.CO;2.

, C.-W. Su, and P. A. McMurtry, 1997: Modeling entrainment and finescale mixing in cumulus clouds. J. Atmos. Sci., 54, 2697-2712, https://doi.org/10.1175/1520-0469(1997)054<2697:MEAFMI> 2.0.CO;2.

Lehmann, K., H. Siebert, and R. A. Shaw, 2009: Homogeneous and inhomogeneous mixing in cumulus clouds: Dependence on local turbulence structure. J. Atmos. Sci., 66, 3641-3659, https://doi.org/10.1175/2009JAS3012.1.
Marshall, J. S., and M. P. Langleben, 1954: A theory of snow-crystal habit and growth. J. Meteor., 11, 104-120, https://doi.org/10.1175/ 1520-0469(1954)011<0104:ATOSCH >2.0.CO;2.

Menon, S., and A. R. Kerstein, 2011: The linear-eddy model. Turbulent Combustion Modeling: Fluid Mechanics and Its Applications, T. Echekki and E. Mastorakos, Eds., Springer, 221-247.

Miller, T. L., and K. C. Young, 1979: A numerical simulation of ice crystal growth from the vapor phase. J. Atmos. Sci., 36, 458-469, https://doi.org/10.1175/1520-0469(1979)036<0458:ANSOIC > 2.0.CO;2.

Mitchell, D. L., 1996: Use of mass-and area-dimensional power laws for determining precipitation particle terminal velocities. J. Atmos. Sci., 53, 1710-1723, https://doi.org/10.1175/15200469(1996)053<1710:UOMAAD>2.0.CO;2.

Morrison, H., G. De Boer, G. Feingold, J. Harrington, M. D. Shupe, and K. Sulia, 2012: Resilience of persistent Arctic mixed-phase clouds. Nat. Geosci., 5, 11-17, https://doi.org/ 10.1038/ngeo1332.

Pruppacher, H. R., and J. D. Klett, 1997: Microphysics of Clouds and Precipitation. 2nd ed. Kluwer Academic, 954 pp.

Shima, S.-I., K. Kusano, A. Kawano, T. Sugiyama, and S. Kawahara, 2009: The super-droplet method for the numerical simulation of clouds and precipitation: A particle-based and probabilistic microphysics model coupled with a non-hydrostatic model. Quart. J. Roy. Meteor. Soc., 135, 1307-1320, https://doi.org/ 10.1002/qj.441.

Shupe, M. D., and Coauthors, 2008: A focus on mixed-phase clouds: The status of ground-based observational methods. Bull. Amer. Meteor. Soc., 89, 1549-1562, https://doi.org/ 10.1175/2008BAMS2378.1.

Solomon, A., G. Feingold, and M. D. Shupe, 2015: The role of ice nuclei recycling in the maintenance of cloud ice in Arctic mixed-phase stratocumulus. Atmos. Chem. Phys., 15, 10631 10643 , https://doi.org/10.5194/acp-15-10631-2015.

Squires, P., 1952: The growth of cloud drops by condensation. I. General characteristics. Aust. J. Sci. Res., 5, 59-86, https:// doi.org/10.1071/CH9520059.

Storelvmo, T., I. Tan, and A. V. Korolev, 2015: Cloud phase changes induced by $\mathrm{CO}_{2}$ warming-A powerful yet poorly constrained cloud-climate feedback. Curr. Climate Change Rep., 1, 288-296, https://doi.org/10.1007/s40641-015-0026-2.

Su, C.-W., S. K. Krueger, P. A. McMurtry, and P. H. Austin, 1998: Linear eddy modeling of droplet spectral evolution during entrainment and mixing in cumulus clouds. Atmos. Res., 47-48, 41-58, https://doi.org/10.1016/S0169-8095(98)00039-8.

Tölle, M. H., and S. K. Krueger, 2014: Effects of entrainment and mixing on droplet size distributions in warm cumulus clouds. J. Adv. Model. Earth Syst., 6, 281-299, https://doi.org/10.1002/ 2012MS000209.

Unterstrasser, S., and I. Sölch, 2014: Optimisation of the simulation particle number in a Lagrangian ice microphysical model. Geosci. Model Dev., 7, 695-709, https://doi.org/10.5194/gmd-7-695-2014.

Wegener, A., 1911: Thermodynamik der Atmosphäre. J. A. Barth, 331 pp.

Yang, F., M. Ovchinnikov, and R. A. Shaw, 2013: Minimalist model of ice microphysics in mixed-phase stratiform clouds. Geophys. Res. Lett., 40, 3756-3760, https://doi.org/10.1002/grl.50700. 